International Journal of Dentistry and Oral Science (JDDOS)

ISSN: 2377-8075

\title{
The Challenge Treatment of Medication Related Osteonecrosis of the Jaw: A Case Report
}

M Rodríguez Sánchez ${ }^{1 *}$, Bassi $\mathrm{AP}^{2}$, Carvalho PS $^{3}$

Case Report

${ }^{1}$ Postgraduate Student, Department of Surgery and Integrated Clinics, Aracatuba Dental School, Sao Paulo State University, Brazil.

${ }^{2}$ Professor at Aracatuba Dental School, Department of Surgery and Integrated Clinics, Sao Paulo State University, Aracatuba SP, Brazil.

${ }^{3}$ Professor at Bauru Dental School, Department of Stomatology, Sao Paulo State University, Bauru SP, Brazil.

\begin{abstract}
Bisphosphonates toxicological effect on alveolar bone could be associated to medication related osteonecrosis of the jaw. (MRONJ) Oral surgical procedures as; tooth extractions, implants placements or trauma are suggested risk factors. The aim of this paper is to describe a case of MRONJ and recurrence after the first right mandibular molar extraction. A 54 years old man was referred to the Department of Surgery and Integrated Clinic of Aracatuba Dental School, Univ. Estadual Paulista - UNESP with a medical history of myeloma multiple and treated with zoledronic acid during 3 years. Her dental history consisted of a mandibular molar extraction 3 years after having finished zoledronic acid intake. After the dental extraction the patient developed a clinical features of MRONJ that was treated with antibiotics, sequestrectomy, hyperbaric oxygen, with apparently total resolution for about five month. The post-operative $6^{\text {th }}$ month the patient complained of recurrent pain, exudates and swelling. Antibiotic were prescribed in addition to surgical debridement. Even though mucosal coverage was achieved during the second surgical procedures, healing occurred without signs of infection but without complete gingival coverage until the second year postoperative. MRONJ is a challenge complication and there is not a gold standard treatment.
\end{abstract}

Keywords: Osteonecrosis of the Jaw; Oral Surgery; Tooth Extraction; Bisphosphonates.

\section{*Corresponding Author:}

Maria Del Pilar Rodríguez Sánchez DDS, MS, PhD student, Aracatuba Dental School, Rua José Bonifácio, 1193, Aracatuba SP,

Brazil, 16015-050.

Tel: 55-18-36363270

E-mail: marypylo@gmail.com

Received: May 27, 2015

Accepted: July 01, 2015

Published: July 06, 2015

Citation: M Rodríguez Sánchez, Bassi AP, Carvalho PS (2015) The Challenge Treatment of Medication Related Osteonecrosis of the Jaw: A Case Report. Int J Dentistry Oral Sci. 2(7), 102-105. doi: http://dx.doi. org/10.19070/2377-8075-1500022

Copyright: M Rodríguez Sánchez ${ }^{\odot}$ 2015. This is an open-access article distributed under the terms of the Creative Commons Attribution License, which permits unrestricted use, distribution and reproduction in any medium, provided the original author and source are credited.

\section{Introduction}

Bisphosphonates (BP) are broadly used for metabolic bone disorders and bone metastasis treatment [1-4]. Medication related osteonecrosis of the jaw (MRONJ) is a known side effect of bisphosphonates use and other antiresorptive or antiangiogenic agents $[5,6]$. Osteonecrosis of the jaw developed for BP represents an estimate prevalence of $0.10 \%$ related to oral antiresorptive agents [7]. MRONJ is especially linked to high dose, long-term treatments and intravenous administration of BP for myeloma multiple, breast and prostate cancer $[1,8-10]$ particularly the use of highly potent nitrogen-bisphosphonate as zolendronate and pamidronate in the case of BP [10-12].

The MRONJ is also known as avascular necrosis of bone or osteodhondritis dissecans. The clinical hallmark is exposed bone in the maxillofacial region for at list 8 weeks without history of head and neck radiation therapy [5], but with previous treatment of antiresorptive or antiangiogenic agents [6]. Some cases are associated to pain, local swelling, signs of infection after any invasive dental procedure or dental prosthesis trauma [5].

MRONJ pathogenesis is still understood, BP inhibits osteoclasts and leads with limit bone-remodeling process additionally the angiogenesis capacity is inhibited, affecting the ability of soft and bone tissue healing [13]. It is believed that MRONJ just appears in the maxillofacial region because of the high turnover of alveolar bone in addition to the outside environment exposition throughout the teeth and periodontal ligament. Any kind of trauma over the alveolar region exposes bone to bacterial flora leading to MRONJ [13].

Patients with need for oral surgery including tooth extraction represents the higher risk factor for MRONJ development among bisphosphonates users [10], this is thought because the risk of bacterial invasion through the open wound after tooth extraction, fact that could be precipitated with periodontal disease. Mandible is the most affected region, especially the molar region [9].

Image findings of MRONJ are not precise and are similar to osteomyelitis, osteoradionecrosis, Paget`s disease and bone metasta- 
sis. Images involve osteosclerosis, osteolysis, dense woven bone, thick lamina dura, failure of bone remodeling after surgical procedures those findings have to be correlated to patient medical history and clinical sings and symptoms of MRONJ [14].

Ideal treatments modalities for MRONJ have not been proposed. Conservative treatments could be performed in those patients without sing of infection [15], surgical procedures could be an option to reduce infection and complications, and nevertheless the total resolution of BONJ is low to succeed [11].

The aim of this paper is to described a case of MRONJ and relapse after molar tooth extraction in a 54 years old man diagnosed with myeloma multiple, treated with zolendronate for three years.

\section{Case Report}

A 54 old white male was referred to the oral clinical surgery of Aracatuba Dental School, UNESP - Univ. Estadual Paulista in March 2010 with the main complains of pain and purulent discharge at the right mandibular area. The patient medical history revealed myeloma multiple diagnosed on 2003. He received chemotherapy, bone marrow transplant and zoledronic acid intake for three years (2003-2006). The patient denied other illness, smoking or abuse of alcohol. Her dental history consisted of extraction of the first right inferior molar on August 2009 due to periodontitis, since then, the area did not heal.

Clinical examination showed bone exposure, purulent discharge and soft tissue swelling at the extraction area (Figure 1). Diagnostic image showed lack of dental alveolar bone healing and bony sequestrum (Figure 2).

\section{Treatment Protocol}

Under general anesthesia, a crestal incision, extended along the gingival sulcus of the teeth next of the defect was carried out, then a vertical incision was made at this level, next a mucoperiosteal flap was raised to remove the necrotic and infected bone with surgical burs (Figure 3). Followed by smoothing of sharp edges. Bone margins were determined until bleeding bone was seen, the adjacent posterior tooth was also removed, because was next to the margin of the sequestrum (Figure 4). Wound closure with complete gingival closure was achieved without tension. Antibiotic therapy with clindamycin and metronidazole were prescribed in addition to 30 sessions of hyperbaric oxygen. Even though wound closure was achieved during the surgical procedure, after the healing period a complete gingival coverage was not maintained. At the sixth month postoperative the patient complains of pulsatile pain, exposed bone as shown in Figure 5 , purulent exudate at the treated area and facial edema. Antibiotic therapy with clindamycin and metronidazole were prescribed. Surgical debridement until bleeding bone was carried out under local anesthesia. Clinical signs and symptoms of infection were not present but recurrence occurred. Complete gingival coverage was achieved until the second year postoperative, at this time just mouth rinses were advised.

\section{Discussion}

Treatment protocol to deal with total resolution of MRONJ in BP users is an ideal that has not being elucidated. On this paper was discussed a clinical case with recurrence after different treatments modalities in a patient who was treated with bisphosphonates for tree years and develop MRONJ after a dental extraction carried out after three years of having finished the treatment.

Figure 1. Clinical aspect of bone exposure after seven month of dental extraction.

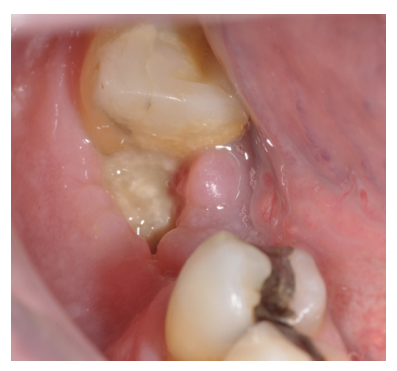

Figure 2. An Orthopantomograph showing a lack of healing after seven month of dental extraction in the mandibular area and bony sequestrum.

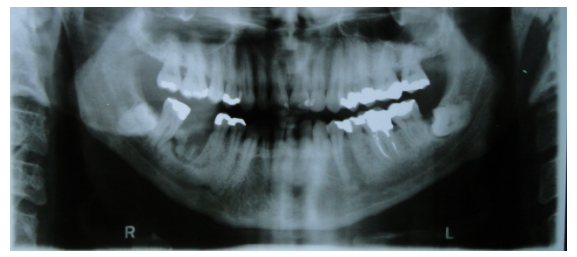

Figure 3. Clinical aspect of the surgical procedure.

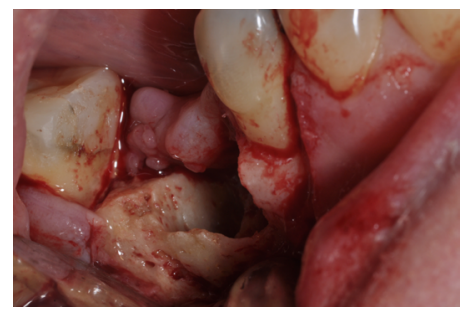


Figure 4. Bony sequestrum and the extracted teeth.

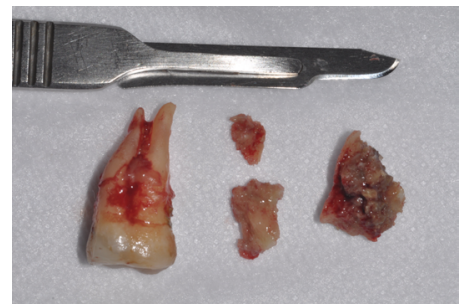

Figure 5. Recurrent bone exposure after 6 months of sequestrectomy.

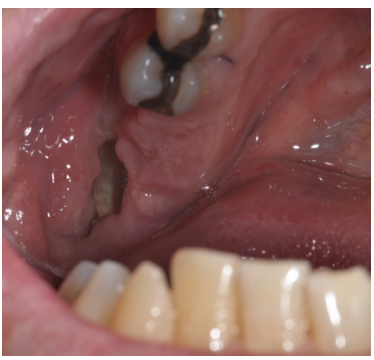

There are recommendations, protocols, and guidelines for MRONJ treatment without clear evidence to support them [6-13, 16-18]. Conservative treatment with the use of mouth rinses and analgesic intake are recommended in AAOMS stage I, which is characterized by necrotic bone exposure without sings of infection [5].

\section{Conservative treatments}

The use of analgesic and antibiotic and mouth rinses are recommended without any surgical management in patients with necrotic bone exposure, pain and signs of infection that is the clinical picture of AAOMS stage II. The isolated microbes have been sensitive to antibiotic therapy with penicillin. Clindamycin, metronidazole, quinolones, doxycycline and erythromycin in are advised in whom are allergic to penicillin $[5,6]$.

Minimal invasive debridement seems suitable in some cases, to reduce the volume of exposed necrotic bone and the biofilm formation around it; this procedure can benefit the antibiotic therapy [6]. Primary mucosal closure with reduce periosteal stripping, when possible offers reliable results, since bone is necrotic and avascular, the overlying mucosa is vascular in patients with AAOMS stage III $[5,7,19]$.

\section{Surgical approach}

Surgical procedures to remove bone sequestrum, decortication, or segmental resection are advised in advance stages of MRONJ (stage III), necrotic bone is debrided until bleeding surgical margins are achieved $[16,18,20]$. Segmental resection contributes to mucosa closure without large manipulation of soft tissue [19]. Even though, sequestrectomy until bleeding margins was carried out and primary mucosal closure without periosteal stripping was achieved during the first surgical procedure, it was not expected to have a MRONJ recurrence after six months on this case.

\section{Adjunctive Therapies}

Adjunctive treatments including laser, growth factors, ozone and hyperbaric oxygen therapy have been proposed, due to the benefits of the reactive oxygen involved in bone turnover during osteoradionecrosis treatment $[17,21,22]$. There is still scarce evidence about the real benefits of the in this case, nonetheless recurrence occur.

$\mathrm{BP}$ holyday treatment in BONJ diagnosis is still controversial; there are no prospective clinical trials to support this treatment modality, it is known the long lasting effects of bisphosphonates in the skeleton, oscillating among one to ten years, however bone metabolism caused by bisphosphonates will not possible decline and provoke clinical changes on MRONJ after holyday treatment for short periods $[1,7]$. The oncologist or patient physician should measure the risk and benefits of bisphosphonates therapy discontinuation [5]. It is important to highlight that in this case zoledronic acid intake was three years previous dental invasive treatment.

ONJ pathophysiology is still understood, we believe, this fact is the key of treatments failure. BPs suppress bone turnover [23], which provoke accumulative bone microdamage and avoid them to repair due to the BPs antiresorptive effect, provoking microdamage accumulation, [24, 25] this effect follow for disruption of mucosa integrity that lead microorganism invasion, and subsequently local infection development $[23,25,26]$ in which macrophages that are cells uncharged of defense are affected by BP use. Macrophages are derived from the same line of monocytes as osteoclasts, we relate this fact with the incapacity of macrophages to react facing the infection [27]. BP has an effect on bone marrow monocytes the macrophages, osteoclast precursors. Macrophages act during inflammatory response $[23,28]$, but in the case of MRONJ they are unable to react facing the local infection, we believe that this last issue is more related to the challenge on MRNJ treatment and the difficult of successful outcomes.

\section{Conclusion}

Management of MRONJ remains a challenge, a complete resolution of MRONJ was not achieved with all treatment modalities proposed and performed on this case, at the first instance; antibiotic, surgical debridement, hyperbaric oxygen were not effective for achieving the desire result. 


\section{References}

[1]. Janovska Z (2012) Bisphosphonate-related osteonecrosis of the jaws. A severe side effect of bisphosphonate therapy. Acta Medica 55(3): 111-115.

[2]. Griz L, Colares V, Bandeira F (2006) Treatment of Paget's disease of bone: importance of the zoledronic acid. Arq Bras Endocrinol Metabol 50(5): 845851.

[3]. Tripathi A, Pandey S, Singh SV, Kumar Sharma N, et al (2011) Bisphosphonate therapy for skeletal malignancies and metastases: Impact on jaw bones and prosthodontic concerns. J Prosthodont 20(7): 601-603.

[4]. Russell RGG (2011) Bisphosphonates: The first 40years. Bone 49(1): 2-19.

[5]. Ruggiero SL, Dodson TB, Assael LA, Landesberg R, Marx RE, et al. (2009) American Association of Oral and Maxillofacial Surgeons position paper on bisphosphonate-related osteonecrosis of the jaws--2009 update. J Oral Maxillofac Surg 67(Suppl 5): 2-12.

[6]. Ruggiero SL, Dodson TB, Fantasia J, Goodday R, Aghaloo T, et al. (2014) American Association of Oral and Maxillofacial Surgeons Position Paper on Medication-Related Osteonecrosis of the Jaw-2014 Update. J Oral Maxillofac Surg 72(10): 1938-1956.

[7]. Hellstein JW, Adler RA, Edwards B, Jacobsen PL, Kalmar JR, et al. (2011) Managing the care of patients receiving antiresorptive therapy for prevention and treatment of osteoporosis: executive summary of recommendations from the American Dental Association Council on Scientific Affairs. J Am Dent Assoc 142(11): 1243-1251.

[8]. Dodson TB (2009) Intravenous Bisphosphonate Therapy and Bisphosphonate-Related Osteonecrosis of the Jaws. J Oral Maxillofac Surg 67(Suppl 5): 44-52.

[9]. Boonyapakorn T, Schirmer I, Reichart PA, Sturm I, Massenkeil G (2008) Bisphosphonate-induced osteonecrosis of the jaws: Prospective study of 80 patients with multiple myeloma and other malignancies. Oral Oncol 44(9): 857-869.

[10]. Kos M (2015) Incidence and risk predictors for osteonecrosis of the jaw in cancer patients treated with intravenous bisphosphonates. Arch Med Sci 11(2): 319-324.

[11]. Kühl S, Walter C, Acham S, Pfeffer R, Lambrecht JT (2012) Bisphosphonate-related osteonecrosis of the jaws - A review. Oral Oncol 48(10): 938947.

[12]. Lobato JV, Mauricio AC, Rodrigues JM, et al. (2008) Jaw avascular osteonecrosis after treatment of multiple myeloma with zoledronate. J Plast Reconstr Aesthet Surg 61(1): 99-106

[13]. McLeod NMH, Brennan PA, Ruggiero SL (2012) Bisphosphonate osteonecrosis of the jaw: A historical and contemporary review. Surgeon 10(1): 36-42.

[14]. Arce K, Assael LA, Weissman JL, Markiewicz MR (2009) Imaging Findings in Bisphosphonate-Related Osteonecrosis of Jaws. J Oral Maxillofac Surg 67(Suppl 5): 75-84.

[15]. Goytia RN, Salama A, Khanuja HS (2009) Bisphosphonates and osteonecrosis: potential treatment or serious complication? Orthop Clin North Am
40(2): 223-234.

[16]. Khan AA, Sandor GK, Dore E, Morrison AD, Alsahli M, et al. (2008) Canadian consensus practice guidelines for bisphosphonate associated osteonecrosis of the jaw. J Rheumatol 35(7): 1391-1397.

[17]. Khan AA, Morrison A, Hanley DA, Felsenberg D, McCauley LK, et al. (2015) Diagnosis and management of osteonecrosis of the jaw: a systematic review and international consensus. J Bone Miner Res 30(1): 3-23.

[18]. Yoneda T, Hagino H, Sugimoto T, Ohta H, Takahashi S, et al. (2010) Bisphosphonate-related osteonecrosis of the jaw: position paper from the Allied Task Force Committee of Japanese Society for Bone and Mineral Research, Japan Osteoporosis Society, Japanese Society of Periodontology, Japanese Society for Oral and Maxillofacial Radiology, and Japanese Society of Oral and Maxillofacial Surgeons. J Bone Miner Metab 28(4): 365-383.

[19]. Markose G, Mackenzie FR, Currie WJ, Hislop WS (2009) Bisphosphonate osteonecrosis: a protocol for surgical management. Br J Oral Maxillofac Surg 47(4): 294-297.

[20]. Khosla S, Burr D, Cauley J, Dempster DW, Ebeling PR, et al. (2007) Bisphosphonate-associated osteonecrosis of the jaw: report of a task force of the American Society for Bone and Mineral Research. J Bone Miner Res 22(10): 1479-1491.

[21]. McLeod NM, Patel V, Kusanale A, Rogers SN, Brennan PA (2011) Bisphosphonate osteonecrosis of the jaw: a literature review of UK policies versus international policies on the management of bisphosphonate osteonecrosis of the jaw. Br J Oral Maxillofac Surg 49(5): 335-342.

[22]. Fliefel R, Troltzsch M, Kuhnisch J, Ehrenfeld M, Otto S (2015) Treatment strategies and outcomes of bisphosphonate-related osteonecrosis of the jaw (BRONJ) with characterization of patients: a systematic review. Int J Oral Maxillofac Surg 44(5): 568-585.

[23]. Serra MP, Llorca CS, Donat FJ (2008) Oral implants in patients receiving bisphosphonates: a review and update. Med Oral Patol Oral Cir Bucal 13(12): E755-760.

[24]. Hoefert S, Schmitz I, Tannapfel A, Eufinger H (2010) Importance of microcracks in etiology of bisphosphonate-related osteonecrosis of the jaw: a possible pathogenetic model of symptomatic and non-symptomatic osteonecrosis of the jaw based on scanning electron microscopy findings. Clin Oral Investig 14(3): 271-284.

[25]. Kwon TG, Lee CO, Park JW, Choi SY, Rijal G, et al. (2014) Osteonecrosis associated with dental implants in patients undergoing bisphosphonate treatment. Clin Oral Implants Res 25(5): 632-640.

[26]. Ruggiero SL, Mehrotra B, Rosenberg TJ, Engroff SL (2004) Osteonecrosis of the jaws associated with the use of bisphosphonates: a review of 63 cases. J Oral Maxillofac Surg 62(5): 527-534.

[27]. Jacobsen C, Metzler P, Rössle M, Obwegeser J, Zemann W, et al. (2013) Osteopathology induced by bisphosphonates and dental implants: clinical observations. Clin oral investig 17(1): 167-175.

[28]. Pazianas M (2011) Osteonecrosis of the jaw and the role of macrophages. J Natl Cancer Inst103(3): 232-240. 